\title{
ASYMPTOTIC PSEUDOMODES OF TOEPLITZ MATRICES
}

\author{
ALbrecht BötTCHer, SERGei GRUdSKY ${ }^{1}$ AND JÉRÉMIE UnTERBERGER
}

\begin{abstract}
Questions in probability and statistical physics lead to the problem of finding the eigenvectors associated with the extreme eigenvalues of Toeplitz matrices generated by Fisher-Hartwig symbols. We here simplify the problem and consider pseudomodes instead of eigenvectors. This replacement allows us to treat fairly general symbols, which are far beyond Fisher-Hartwig symbols. Our main result delivers a variety of concrete unit vectors $x_{n}$ such that if $T_{n}(a)$ is the $n \times n$ truncation of the infinite Toeplitz matrix generated by a function $a \in L^{1}$ satisfying mild additional conditions and $\lambda$ is in the range of this function, then $\left\|T_{n}(a) x_{n}-\lambda x_{n}\right\| \rightarrow 0$.
\end{abstract}

Mathematics subject classification (2000): 47B35, 15A18, 41A80, 46N30.

Keywords and phrases: Toeplitz matrix, Fisher-Hartwig symbol, eigenvector, pseudomode, fractional Brownian motion.

\section{REFERENCES}

[1] M. N. BARber AND M. E. Fisher, Critical Phenomena in Systems of Finite Thickness, I. The Spherical Model, Annals of Physics 77 (1973), 1-78.

[2] J. G. Brankov, D. M. DancheV, AND N. S. ToncheV, Theory of critical phenonmena in finite-size systems, Series in Modern Condensed Matter Physics Vol. 9, World Scientific, Singapore (2000).

[3] T. H. BERLin AND M. KAC, The spherical model of a ferromagnet, Phys. Rev. 86 (1952), 821-835.

[4] A. BötTCher, M. EMBree, AND L. N. TREFETHEN, Piecewise continuous Toeplitz matrices and operators: slow approach to infinity, SIAM J. Matrix Analysis Appl. 24 (2002), 484-489.

[5] A. BÖTTCHER AND S. GRUDSKY, Toeplitz matrices with slowly growing pseudospectra, In: Factorization, Singular Operators and Related Problems in Honour of Georgii Litvinchuk (S. Samko, A. Lebre, A. F. dos Santos, eds.), pp. 43-54, Kluwer Academic Publishers, Dordrecht 2003.

[6] A. BÖTTCHER AND S. GRUDSKY, Asymptotically good pseudomodes for Toeplitz matrices and WienerHopf operators, Oper. Theory Adv. Appl. 147 (2004), 175-188.

[7] A. BötTCher And B. Silbermann, Toeplitz matrices and determinants with Fisher-Hartwig symbols, J. Funct. Anal. 63 (1985), 178-214.

[8] A. BÖtTCHER AND J. VIRTANEN, Norms of Toeplitz matrices with Fisher-Hartwig symbols, SIAM J. Matrix Analysis Appl. 29 (2007), 660-671.

[9] I. Gohberg AND I. A. Feldman, Convolution Equations and Projection Methods for Their Solution, Amer. Math. Soc., Providence 1974.

[10] M. KAC, W. L. MURDOCK, AND G. SZEGÖ, On the eigenvalues of certain Hermitian forms, J. Rational Mech. Anal. 2 (1953), 767-800.

[11] S. V. PARTER, Extreme eigenvalues of Toeplitz forms and applications to elliptic difference equations, Trans. Amer. Math. Soc. 99 (1961), 153-192.

[12] S. V. PARTER, On the extreme eigenvalues of Toeplitz matrices, Trans. Amer. Math. Soc. 100 (1961), 263-276.

[13] L. REICHEL AND L. N. TREFETHEN, Eigenvalues and pseudo-eigenvalues of Toeplitz matrices, Linear Algebra Appl. 162/164 (1992), 153-185.

[14] G. SAMORODNITSKY AND M. TAQQU, Stable Non-Gaussian Random Processes, Chapman and Hall, New York (1994).

[15] S. SERRA, On the extreme spectral properties of Toeplitz matrices generated by $L^{1}$ functions with several minima/maxima, BIT 36 (1996), 135-142. 
[16] S. SERRA, On the extreme eigenvalues of Hermitian (block) Toeplitz matrices, Linear Algebra Appl. 270 (1998), 109-129.

[17] L. N. TREFETHEN AND M. EMBReE, Spectra and Pseudospectra, The Behavior of Nonnormal Matrices and Operators, Princeton University Press, Princeton 2005.

[18] H. WIDOM, On the eigenvalues of certain Hermitian operators, Trans. Amer. Math. Soc. 88 (1958), 491-522.

[19] N. L. ZAMARASHKIN AND E. E. TYRTYSHNIKOV, Distribution of the eigenvalues and singular numbers of Toeplitz matrices under weakened requirements on the generating function, Sb. Math. 188 (1997), $1191-1201$.

[20] A. Zygmund, Trigonometric Series, Vol. I, University of Cambridge Press, Cambridge 1959. 